\title{
Caracterização da fauna de moscas-das-frutas (Diptera: Tephritidae) na região de Ponta Grossa, Paraná, Brasil
}

\author{
Characterization of the fauna of fruit flies (Diptera: Tephritidae) in the region of Ponta Grossa, \\ Paraná, Brazil
}

\author{
Patricia Elizabeth Husch ${ }^{\text {I }}$ Julianne MilléoII Daniel SedorkoII Ricardo Antonio Ayub ${ }^{\text {II }}$ \\ Domingos Sávio Nunes ${ }^{\text {II }}$
}

RESUMO

Apesar do incentivo e do investimento na produção de frutas no estado do Paraná, pouca informação está disponível sobre as moscas-das-frutas, uma das principais pragas da fruticultura. O presente trabalho teve os objetivos de registrar pela primeira vez a fauna de tefritídeos, na região de Ponta Grossa, e caracterizar a sua comunidade por meio de diferentes índices faunísticos. Adultos de tefritídeos foram coletados por meio de frascos caça-moscas com atrativos alimentares em quatro pomares de agosto a dezembro de 2009. Foi coletado um total de 2.428 tefritídeos distribuídos em sete espécies: Anastrepha fraterculus Wiedemann, Anastrepha montei Lima, Anastrepha pseudoparallela Loew, Anastrepha sororcula Zucchi, Ceratitis capitata Wiedemann, Rhagoletis sp. Loew e Hexachaeta sp. Loew. A. fraterculus foi a única espécie comum aos quatro pomares, ocorrendo com frequência acima de $80 \%$. Este é o primeiro registro de A. montei no Paraná. A. fraterculus, A. sororcula e C. capitata apresentaram os maiores índices de frequência, abundância, dominância e constância, sendo, portanto, consideradas espécies predominantes. O maior índice de diversidade $\left(H^{\prime}=0,4360\right)$ ocorreu no pomar 2 , diferindo significativamente dos demais pomares (teste $t, P<0,05)$. Os valores do índice de Margalef foram baixos (próximos ou inferiores a 0,5), confirmando haver predominância de espécies. Quanto à similaridade de espécies entre os pomares, determinada pelo coeficiente de Jaccard e índice de BrayCurtis, o pomar 4, onde ocorreram apenas duas espécies $(96 \%$ de A. fraterculus e $4 \%$ de A. pseudoparallela) forma um agrupamento distinto dos demais pomares amostrados, no qual ocorreram quatro espécies de tefritídeos.

Palavras-chave: análise faunística, tefritídeos, fruticultura, Campos Gerais do Paraná.

\begin{abstract}
In spite of encouragement and investment in fruit production in the state of Paraná, little information is available on the fruit flies, a major pest in fruit growing. The present study had the objective of recording for the first time the tephritid fauna in the region of Ponta Grossa, and characterize the insect community through different faunal indices. Adult tephritids were collected by means of flapper bottles with food baits in four orchards from August to December 2009. A total of 2,428 tephritids distributed in seven species were collected: Anastrepha fraterculus Wiedemann, Anastrepha montei Lima, Anastrepha pseudoparallela Loew, Anastrepha sororcula Zucchi, Ceratitis capitata Wiedemann, Rhagoletis sp. Loew and Hexachaeta sp. Loew. A. fraterculus was the only species common to the four orchards, occurring in a frequency higher than $80 \%$. This is the first record of $\boldsymbol{A}$. montei in the state of Paraná. A. fraterculus, $\boldsymbol{A}$. sororcula and $\boldsymbol{C}$. capitata had the highest frequency, abundance, dominance and constancy, and are therefore considered predominant species. The highest diversity index $\left(H^{\prime}=0.4360\right)$ occurred in the orchard 2, differing significantly from all orchards ( $t$ test, $P<0.05)$. The Margalef index values were low (close to or below 0.5) confirming that there are predominant species. Regarding the similarity of species between the orchards, that was determined by the coefficient of Jaccard and the Bray-Curtius index, the orchard 4, where occurred only two species (96\% of A. fraterculus and $4 \%$ of A. pseudoparallela), forms a distinct group from other orchard sampled, where there were four species of Tephritidae.
\end{abstract}

Key words: faunistic analysis, Tephritidae, fruit production, Campos Gerais do Paraná.

IPrograma de Pós-graduação em Entomologia, Departamento de Zoologia, Universidade Federal do Paraná (UFPR), CP 19020, 81531-980, Jardim das Américas, Curitiba, PR, Brasil. E-mail: patihusch@gmail.com. Autor para correspondência.

${ }^{\mathrm{II}}$ Universidade Estadual de Ponta Grossa (UEPG), Ponta Grossa, PR, Brasil. 


\section{INTRODUÇÃO}

O termo "mosca-das-frutas" é a designação comum das espécies de dípteros da família Tephritidae, visto que suas larvas se alimentarem da polpa dos frutos de diferentes espécies nativas e cultivadas. Após a oviposição, as fêmeas deixam um orifício que serve de porta de entrada para microorganismos que causam o apoderecimento do fruto. Soma-se a esses danos, o fato de as moscas-das-frutas constituirem pragas quarentenárias para alguns países importadores de frutas brasileiras. Portanto, os tefritídeos apresentam grande importância como praga de fruteiras cultivadas. No Brasil, os tefritídeos relatados como causadores de danos em pelo menos uma cultura estão distribuídos em quatro gêneros: Anastrepha Schiner, Bactrocera Macquart, Ceratitis MacLeay e Rhagoletis Loew (ZUCCHI, 2000a).

Apesar do incentivo e do investimento na produção de frutas no estado do Paraná, existem poucos trabalhos referentes aos tefritídeos nesse estado. Os primeiros registros de espécies são de FEHN (1977; 1981) para os municípios de Curitiba e Irati, na região metropolitana, destacando a ocorrência de Anastrepha fraterculus e Ceratitis capitata em pomares de pêssego (Prunus persica). Somente 25 anos depois surgiram novos registros, que foram feitos por ALBUQUERQUE et al. (2002), citando a ocorrência daquelas mesmas espécies de tefritídeos em pomar de acerola (Malphigia glabra) no município de Ângulo, na região de Maringá. Com base em coleções do Paraná, GARCIA (2003) citou a ocorrência de nove espécies de Anastrepha no estado.

Não existem informações sobre as espécies de moscas-das-frutas que ocorrem em Ponta Grossa. Apesar de ser tradicional na produção de grãos, anualmente, vêm crescendo os investimentos em fruticultura na região, que, atualmente, conta com pomares de maçã (Malus sp.) e caqui (Diospyros kaki) para exportação. De acordo com os relatórios da Secretaria da Agricultura e do Abastecimento do Paraná de 2006 e 2007 (SEAB, 2009), a região destaca-se também na produção de laranja (Citrus sinensis), melancia (Citrullus lanatus), pêra (Pyrus communis), tangerina (Citrus reticulata), uva (Vitis sp.) e pêssego. Todas essas frutas foram registradas como hospedeiras de larvas de tefritídeos em outros estados brasileiros (ZUCCHI, 2000b; 2001). Nesse contexto, o presente trabalho teve como objetivos registrar a assembleia de tefritídeos na região de Ponta Grossa, PR, e caracterizála em pomares de pêssego por meio de diferentes índices faunísticos.

\section{MATERIAL E MÉTODOS}

Os adultos de tefritídeos foram capturados por meio de armadilhas do tipo frasco caça-moscas com atrativo alimentar, sendo distribuídas em quatro pomares localizados na região de Ponta Grossa, PR: Pomar 1 - propriedade Santo Antônio $\left(8.000 \mathrm{~m}^{2}\right)$, em Guaragi (25 13 ' S; 50 $52^{\circ}$ 'O), distrito de Ponta Grossa, com $886 \mathrm{~m}$ de altitude e plantio de pessegueiro com finalidade comercial (variedades Chimarrita e Texano). Este pomar localiza-se em uma área rural e possui em seu entorno cultivo de soja, além de fragmentos da vegetação secundária da Floresta Ombrófila Mista; Pomar 2 - propriedade Cinto Verde (cerca de $10.000 \mathrm{~m}^{2}$ ), no bairro de Uvaranas ( $\left.25^{\circ} 6^{\prime} \mathrm{S} ; 50^{\circ} 5^{\prime} \mathrm{O}\right)$, Ponta Grossa, com $857 \mathrm{~m}$ de altitude e plantio de pessegueiro, com finalidade comercial (variedades Chimarrita, Charme e Eragil). Este pomar localiza-se em uma área urbana e, em seu entorno, há cultivo de soja no período de coleta das moscas, além de pequenos fragmentos de vegetação secundária da Floresta Ombrófila Mista; Pomar 3 - pomar da Estação Experimental de Ponta Grossa/IAPAR $\left(18.000 \mathrm{~m}^{2}\right)$, na Rodovia do Café, km 496 (25ㅇ's; 509'O), em Ponta Grossa, com 869m de altitude e plantio de pessegueiro e ameixeira (Prunus serrulata) (com mais de 40 variedades comerciais e não comerciais). Este pomar possui, em seu entorno, cultivo de soja e feijão, além de fragmentos de vegetação secundária da Floresta Ombrófila Mista e áreas de reflorestamento com eucalipto; Pomar 4 - pomar do Colégio Agrícola Estadual Augusto Ribas - CAAR (com cerca de $1.000 \mathrm{~m}^{2}$ ), no bairro de Uvaranas, Ponta Grossa (255's; 506' W), que tem, como finalidade didática, $904 \mathrm{~m}$ de altitude e plantio de pessegueiro, caquizeiro, pereira, macieira, laranjeira, tangerineira, tangerineira poncã (C. reticulata, variedade poncã), limoeiro (Citrus limonium) e nectarineira (P. persica, variedade nucipersica). Este pomar localiza-se em área urbana, com fragmentos de campo ao redor, além de cultivo de hortaliças.

Segundo CRUZ (2007), o clima da região é do tipo $\mathrm{Cfb}$, temperado, com temperaturas médias anuais variando entre $17^{\circ} \mathrm{Ce} 21^{\circ} \mathrm{C}$; pluviosidade média em torno de 1.600 a $1.800 \mathrm{~mm}$ anuais, sendo o mês de janeiro o mais chuvoso e agosto o menos chuvoso, sem estação seca definida, e umidade relativa média do ar de 75 a $80 \%$; com ventos suaves e moderados entre

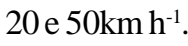

Foram instaladas quatro armadilhas por pomar, sendo, no pomar 1, armadilhas modelo McPhail e, nos pomares 2, 3 e 4, as armadilhas confeccionadas com garrafas PET, ambas contendo $300 \mathrm{~mL}$ de solução 
atrativa, seguindo metodologia descrita em AGUIARMENEZES et al. (2006). Nos pomares 1 e 2, o atrativo utilizado foi proteína hidrolisada a $5 \%$ e, nos pontos 3 e 4 , foi suco de uva diluído a $25 \%$. As armadilhas foram instaladas na periferia dos pomares, fixadas a uma altura de dois metros do solo (aproximadamente $3 / 4$ da altura da árvore), sendo distanciadas de 20 a 50 metros entre elas, como proposto por AGUIAR-MENEZES et al. (2006), visto que três, dos quatro pomares, têm finalidades comerciais e experimentais, as armadilhas também atuaram na interceptação das moscas, diminuindo a sua entrada para os pomares. A diferença no uso de armadilhas e atrativos foi devido ao aproveitamento do material disponível nos pomares comerciais, visto que não foram encontradas diferenças significativas entre eles como nos trabalhos realizados por SCOZ et al. (2006).

Os adultos capturados nas armadilhas foram coletados semanalmente durante o período de frutificação do pêssego (de setembro a dezembro de 2009, com exceção dos dias 03 e 17 de novembro), totalizando 13 coletas. Os insetos coletados foram armazenados em potes plásticos com álcool hidratado a $75 \%$ e etiquetados para posterior triagem no laboratório. Na triagem, os tefritídeos foram separados dos demais insetos e, em seguida, separados por sexo e morfoespécies e contados. Para identificação das espécies de tefritídeos, foram adotadas as chaves dicotômicas de ZUCCHI (2000a) e URAMOTO (2002) para o gênero Anastrepha. De acordo com tais referências, a identificação das espécies de Anastrepha só é possível pelas características morfológicas da fêmea, principalmente do acúleo. E, portanto, apenas os exemplares identificados especificamente foram incluídos nas análises dos índices faunísticos. Esses insetos encontram-se depositados na Coleção Entomológica dos Campos Gerais do Paraná (CECG), Departamento de Biologia Geral, Universidade Estadual de Ponta Grossa (UEPG), Ponta Grossa, PR.

Os dados de coleta foram submetidos à análise faunística pelo programa ANAFAU (MORAES et al., 2003), no qual foram avaliados os índices de frequência, abundância, dominância e constância. As espécies que apresentaram os maiores valores desses índices foram consideradas espécies predominantes no local. Na classificação do ANAFAU, é adicionada a classe extrema (super), referente aos valores discrepantes de número de insetos, discriminados através da análise de resíduos. Esses valores não são considerados no cálculo dos parâmetros de dominância, frequência e abundância. A diversidade de tefritídeos nos pomares foi calculada pelo índice de Shannon-Wiener (H') e, para verificar se existiu diferença significativa entre os resultados, foi aplicado o teste $t$. O índice de riqueza de espécies adotado foi de Margalef $(\alpha)$ e, para estimar a uniformidade em termos de abundância de indivíduos entre as espécies da comunidade amostrada, foi calculado o índice de equitabilidade (E'). Para avaliação da semelhança entre as populações dos quatro pomares, foram aplicados o coeficiente de Jaccard, que indicou a similaridade entre os pomares quanto à composição das espécies (dados de presença/ausência), e o índice de Bray-Curtis, que indicou a relação entre os pomares quanto à estrutura de comunidade (dados de abundância). Essas análises foram realizadas pelo software PAST versão 2.07 (HAMMER et al., 2001).

\section{RESULTADOS E DISCUSSÃO}

Foi coletado um total de 2.428 tefritídeos (1497 e $931 \precsim$ ) nos quatro pomares amostrados. Excetuando os machos de Anastrepha, obteve-se um total de 1.503 tefritídeos (Tabela 1). Todos exemplares pertenceram à subfamília Trypetinae, sendo distribuídos em sete espécies: quatro do gênero Anastrepha (A. fraterculus; A. montei; A. pseudoparallela; e A. sororcula), Ceratitis capitata, uma do gênero Hexachaeta; e uma do gênero Rhagoletis. Esse é o primeiro registro de A. montei no estado do Paraná, onde os demais gêneros/espécies já foram registrados (GARCIA; 2003; ZUCCHI, 2007), sendo que $\boldsymbol{A}$. fraterculus e $\boldsymbol{C}$. capitata já tem ocorrência registrada em pomares de pêssego nesse estado(FEHN, 1977; 1982).

Anastrepha fraterculus foi a única espécie comum aos quatro pomares, representando $95,54 \%$ do total capturado e a única classificada como super frequente, super dominante, super abundante e constante nas amostragens, sendo capturada em 12 das 13 coletas realizadas (Tabela 1). FEHN (1977; 1982) também a considerou a mais abundante em pomares de pêssego em dois municípios paranaenses (Curitiba e Irati). Esta espécie, devido ao seu hábito generalista e ação competitiva, provavelmente, está afastando as outras espécies do local. Esses dados corroboram as afirmações de MALAVASI et al. (2000), que indicam a espécie A. fraterculus como praga primária de maior importância na Argentina, Uruguai e nos estados do sul e sudeste do Brasil. A relevância de A. fraterculus neste trabalho confirma a afirmação de AGUIARMENEZES et al. (2008) de que uma ou duas espécies de tefritídeos são predominantes, particularmente em pomares comerciais homogêneos onde normalmente constituem praga-chave. 
Tabela 1 - Análise faunística da fauna de tefritídeos em quatro pomares na região de Ponta Grossa, PR, de setembro a dezembro/2009.

\begin{tabular}{|c|c|c|c|c|c|c|c|c|c|}
\hline Espécie & Pomar 1 & Pomar 2 & Pomar 3 & Pomar 4 & Total & $\mathrm{F}^{(1)}$ & $\mathrm{C}^{(2)}$ & $\mathrm{Do}^{(3)}$ & $\mathrm{A}^{(4)}$ \\
\hline A. fraterculus & 361 & 273 & 689 & 113 & 1436 & sf & $\mathrm{w}$ & $\mathrm{sd}$ & $\mathrm{sa}$ \\
\hline A. sororcula & 5 & 19 & 7 & 0 & 31 & $\mathrm{mf}$ & $\mathrm{w}$ & $\mathrm{d}$ & $\mathrm{ma}$ \\
\hline A. montei & 0 & 0 & 2 & 0 & 2 & $\mathrm{f}$ & $\mathrm{z}$ & nd & $\mathrm{c}$ \\
\hline A. pseudoparalella & 0 & 0 & 0 & 5 & 5 & $\mathrm{f}$ & $\mathrm{y}$ & nd & $\mathrm{c}$ \\
\hline C. capitata & 5 & 14 & 6 & 0 & 25 & $\mathrm{mf}$ & $\mathrm{w}$ & $\mathrm{d}$ & $\mathrm{a}$ \\
\hline Rhagoletis sp. & 3 & 0 & 0 & 0 & 3 & $\mathrm{f}$ & z & nd & $\mathrm{c}$ \\
\hline Hexachaeta sp. & 0 & 1 & 0 & 0 & 1 & $\mathrm{f}$ & z & nd & $\mathrm{c}$ \\
\hline Total & 374 & 307 & 704 & 118 & 1503 & & & & \\
\hline $\mathrm{H}^{,(5)}$ & 0,1882 & 0,4360 & 0,1242 & 0,1754 & 0,2368 & & & & \\
\hline$\alpha^{(6)}$ & 0,5064 & 0,5238 & 0,4575 & 0,2096 & 0,8202 & & & & \\
\hline$E^{(7)}$ & 0,1358 & 0,3145 & 0,0896 & 0,2531 & 0,1217 & & & & \\
\hline
\end{tabular}

${ }^{(1)}$ Frequência $(\mathrm{F})=$ pouco frequente $(\mathrm{pf})$, frequente $(\mathrm{f})$, muito frequente $(\mathrm{mf})$, super frequente $(\mathrm{sf})$. ${ }^{(2)} \mathrm{Constância}(\mathrm{C})=\mathrm{constante}(\mathrm{w})$ - mais de $50 \%$ das coletas, acessória (y) - entre 25 a $50 \%$ das coletas; acidental (z) - menos de $25 \%$ das coletas. ${ }^{(3)}$ Dominância (Do) $=$ não dominante (nd) - espécies cuja frequência é menor que o limite de dominância, dominante (d) - espécies cuja frequência é maior que o limite de dominância, super dominante $(\mathrm{sd}) .{ }^{(4)}$ Abundância $(\mathrm{A})=$ raro $(\mathrm{r})-\mathrm{n}^{\mathrm{o}}$ indivíduos menor que o limite inferior do IC a $1 \%$ de probabilidade, disperso (d) - $\mathrm{n}^{\mathrm{o}}$ de indivíduos situado entre os limites inferiores do IC a $5 \%$ e $1 \%$ de probabilidade, comum (c) - $\mathrm{n}^{\mathrm{o}}$ de indivíduos situado dentro do IC a 5\% de probabilidade, abundante (a) $\mathrm{n}^{\circ}$ de indivíduos situado entre os limites superiores do IC a 5\% e $1 \%$ de probabilidade, muito abundante (ma) - $\mathrm{n}^{\mathrm{o}}$ de indivíduos maior que o limite superior do IC a $5 \%$ de probabilidade, super abundante (sa). ${ }^{(5)}$ Índice de Shannon-Wiener (H'). ${ }^{(6)}$ Índice de Margalef $(\alpha){ }^{(7)}$ Índice de equitabilidade (E').

A espécie A. sororcula ocorreu em três pomares, representando $2,06 \%$ do total de exemplares coletados, sendo categorizada como muito frequente, dominante, muito abundante e constante nas amostragens. Essa espécie é praga de importância econômica no Brasil, atacando principalmente frutos de goiaba (Psidium guayava) e outras mirtáceas (ZUCCHI, 2000b). Apesar de amplamente disseminada no país, $\boldsymbol{A}$. sororcula não costuma predominar nos levantamentos. Teve destaque nos trabalhos de AGUIAR-MENEZES et al. (2008) sendo a espécie mais frequente e constante em São João da Barra, RJ, e, no de GUIMARÃES et al. (2008), A. sororcula e $\boldsymbol{A}$. zenildae predominaram em pomares de goiaba no município de Juazeiro do Norte, CE.

Ceratitis capitata $(1,66 \%)$ foi capturada nos pomares 1, 2 e 3 e classificada como muito frequente, dominante, abundante e constante nas amostragens. FEHN (1981) registrou a presença dessa espécie em pomares de pessegueiro no Paraná, nos municípios de Araucária, Campo Largo e Piraquara.

As quatro espécies restantes $(0,74 \%$ do total) foram categorizadas como frequentes, não dominantes, comuns e acidentais na amostragem. Cada uma foi exclusiva em um diferente local de coleta: $\boldsymbol{A}$. montei no pomar 3; A. pseudoparallela no pomar 4; Rhagoletis sp. no pomar 1 e Hexachaeta sp. no pomar 2. A. montei também foi classificada como acidental nas amostragens em pomares de laranja, pêssego e maracujá (Passiflora edulis) por ALBERTI et al. (2009) eZILLI \& GARCIA (2010) em Santa Catarina; e como acessória em matas nativas e pomares domésticos de dois municípios de Tocantins por BOMFIM et al. (2007).

Anastrepha fraterculus e A. sororcula, seguidas por $\boldsymbol{C}$. capitata, por apresentarem maiores índices faunísticos, são apontadas como predominantes, indicando que apresentam grande capacidade adaptativa nesses pomares. Conforme argumentado em AGUIAR-MENEZES et al. (2008), $\boldsymbol{A}$. fraterculus e C. capitata infestam pêssegos em pomares localizados no Paraná (FEHN, 1977; 1981), portanto esses tefritídeos tem grande potencial de ser tornarem pragas dos pessegueiros na região dos Campos Gerais.

Em relação à abundância de moscas-dasfrutas nos quatro pomares amostrados, a sequência em ordem decrescente nos pomares foi: $3(47 \%)>1$ $(25 \%)>2(20 \%)>4(8 \%)$. A grande quantidade de tefritídeos encontrada no pomar 3 (Estação Experimental de Ponta Grossa/IAPAR) pode ser justificada, pois, além de ser a maior área de plantio, no entorno do pomar, existem diversas espécies de frutíferas nativas consideradas hospedeiras desses insetos-pragas.

O pomar 2 apresentou o maior índice de diversidade de Shannon-Wiener (0,4360), mostrando uma diferença significativa em relação aos demais pomares (teste $t, \mathrm{P}<0,05)$ (Tabela 2). Este índice revela 
Tabela 2 - Valores de $t$ e p na comparação dos índices de diversidade de Shannon-Wiener da fauna de tefritídeos entre quatro pomares na região de Ponta Grossa, PR (setembro a dezembro/2009).

\begin{tabular}{|c|c|c|c|c|c|c|}
\hline & $t$ & $\mathrm{p}$ & $t$ & $\mathrm{p}$ & $t$ & $\mathrm{p}$ \\
\hline Pomar 1 & - & - & - & - & - & - \\
\hline Pomar 2 & 3,6767 & $0,00025 *$ & - & - & - & - \\
\hline Pomar 3 & 1,2569 & 0,20924 & 5,257 & $0,00001 *$ & - & - \\
\hline Pomar 4 & 0,1820 & 0,85567 & 3,317 & $0,00101 *$ & 0,7694 & 0,44272 \\
\hline
\end{tabular}

*Resultados significativos $(\mathrm{P}<0,05)$.

que a comunidade de tefritídeos no ponto 2 foi a mais diversa. Todavia, o índice de Shannon-Wiener da região foi baixo (0,2368). SILVEIRANETOet al. (1976) explicam que os valores dos índices de diversidade tendem a serem baixos em locais onde os fatores limitantes e a competição interespecífica atuam intensamente. Nesses locais, as espécies mais comuns aumentam suas populações e as espécies raras apresentam baixo nível populacional. Os resultados aqui obtidos corroboram essa afirmativa, pois mostram a ocorrência de poucas espécies de tefritídeos, mas muitos indivíduos de $\boldsymbol{A}$. fraterculus. Esses valores são em consequência da alta frequência desses insetos, revelando uma espécie dominante, como colocado por URAMOTO (2002).

A riqueza das espécies, medida pelo índice de Margalef, apresentou a seguinte sequência entre os pomares: $2>1>3>4$, com valores próximos ou inferiores a 0,5 , confirmando haver predominância de espécies, ou seja, ocorrência de poucas espécies com populações de muitos indivíduos, com destaque para A. fraterculus, que correspondeu a mais de $80 \%$ dos exemplares capturados em cada pomar. Esses resultados correspondem ao encontrado por outros autores em condições de pomares homogêneos (ALUJA et al., 1996; GARCIA et al., 2003; AGUIARMENEZES et al., 2008). O índice de equitabilidade revela uma maior uniformidade na distribuição das espécies de tefritídeos no pomar 2, seguido dos pomares 4, 1 e 3 . Todavia, os valores estando mais próximos a zero são indicativos da predominância de $\boldsymbol{A}$. fraterculus. Pelo coeficiente de Jaccard (Figura 1a), a comunidade de tefritídeos mostrou $60 \%$ de similaridade entre os pomares 1, 2 e 3; e, isoladamente, ficou o pomar 4 . Esse coeficiente une os locais com a mesma riqueza de espécies, separando-os do pomar 4, onde só duas espécies foram coletadas. O dendrograma de similaridade obtido pelo índice de Bray-Curtis (Figura 1b) mostra que a estrutura da comunidade de tefritídeos nos pomares 1 e 2 apresenta cerca de $83 \%$ de similaridade e forma um agrupamento com o pomar 3 .
Já o pomar 4 apresenta-se distinto dos demais locais amostrados. A maior similaridade entre os pomares 1, 2 e 3 deve-se ao fato de que este índice avalia a proporcionalidade das espécies, de modo que o grande número de $\boldsymbol{A}$. fraterculus e $\boldsymbol{A}$. sororcula coletadas nesses pomares causou maior peso na análise.

A separação do pomar 4, nos dois dendrogramas, indica uma fauna de tefritídeos diferenciada dos demais pomares, o que pode ser atribuído a dois fatores: menor dimensão da área e maior variedade de plantas hospedeiras, sendo que, nos demais pomares, predomina o plantio de pêssego.

\section{CONCLUSÃO}

$\mathrm{Na}$ região de Ponta Grossa, foram encontradas sete espécies de tefritídeos, sendo quatro do gênero Anastrepha (A. fraterculus, A. montei, A. pseudoparallela e A. sororcula), Ceratitis capitata, Rhagoletis sp. e Hexachaeta sp., e o primeiro registro de A. montei no estado do Paraná. De acordo com os resultados encontrados neste trabalho, a comunidade de tefritídeos nos quatro pomares da região de Ponta Grossa caracteriza-se pela predominância de $\boldsymbol{A}$. fraterculus, sendo seguida por A. sororcula e $\boldsymbol{C}$. capitata, as quais apresentam os maiores índices de frequência, abundância relativa, dominância e constância.

\section{AGRADECIMENTOS}

Ao $\mathrm{CNPq}$, pela bolsa de Iniciação Científica para desenvolvimento deste trabalho. À prof ${ }^{a}$ Dra. Keiko Uramoto, Depto. de Genética e Biologia Evolutiva da USP, pelo auxílio na identificação dos exemplares. Ao diretor técnico Jail Bueno (CAAR), ao pesquisador Roberto Hauagge e ao técnico Ozeas Gonçalves (IAPAR) e aos produtores Eloir Moresco e Amarildo Antônio Pramio, por permitirem o desenvolvimento desta pesquisa nos pomares. À Regina Célia Botequio de Moraes, Depto. de Entomologia da ESALQ, pelo envio do programa ANAFAU. 


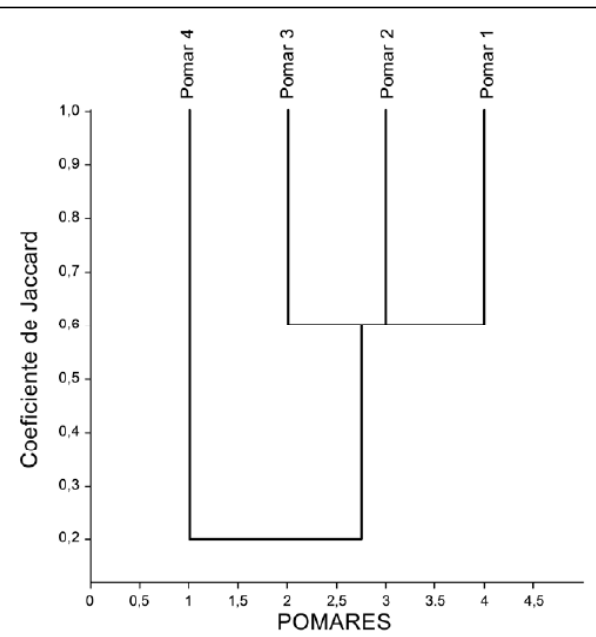

(a)

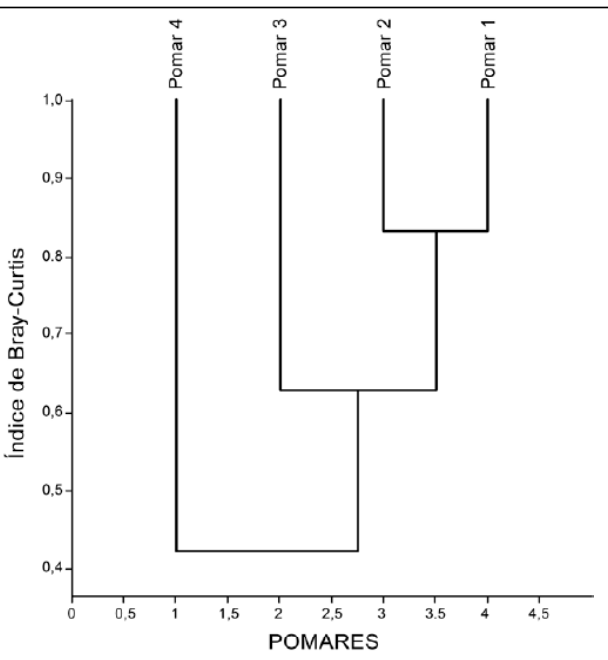

(b)

Figura 1 - Dendrogramas de similaridade entre os quatro pomares quanto à composição de espécies de tefritídeos (região de Ponta Grossa, PR, setembro a dezembro/2009). (a) Coeficiente de Jaccard, dados de presença/ausência de tefritídeos; (b) Índice de Bray-Curtis, dados de abundância de tefritídeos.

\section{REFERÊNCIAS}

AGUIAR-MENEZES, E.L. et al. Armadilha PET para captura de adultos de moscas-das-frutas em pomares comerciais e domésticos. Seropédica, Rio de Janeiro, Brasil. Embrapa Agrobiologia, 2006. 8p. (Circular Técnica n.16). Disponível em: <http://cnpab.embrapa.br/publicacoes/download/ cit016.pdf>. Acesso em: 20 set. 2010.

AGUIAR-MENEZES, E.L. et al. Análise faunística de moscasdas-frutas (Diptera: Tephritidae) nas regiões norte e noroeste do estado do Rio de Janeiro. Neotropical Entomology, v.37, n.1, p.8-14, 2008. Disponível em: <http://www.scielo.br/

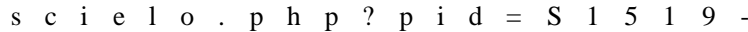
$566 X 2008000100002 \&$ script $=$ sci_arttext $>$. Acesso em: 24 dez. 2011. doi: http://dx.doi.org/10.1590/S1519566X2008000100002.

ALBERTI, S. et al. Moscas-das-frutas em pomares de pessegueiro e maracujazeiro, no município de Iraceminha, Santa Catarina, Brasil. Ciência Rural, v.39, n.5, p.1565-1568, 2009. Disponível em: <http://www.scielo.br/scielo.php?pid=S0103$84782009000500041 \&$ script $=$ sci_arttext $>$. Acesso em: $24 \mathrm{dez}$. 2011 doi: http://dx.doi.org/10.1590/S010384782009005000077 .

ALBUQUERQUE, F.A. et al. Insetos associados à cultura da aceroleira (Malpighia glabra L.) na região de Maringá, Paraná. Acta Scientiarum, Agronomy, v.24, n.5, p.1245-1249, 2002. Disponível em: <http://periodicos.uem.br/ojs/index.php/ ActaSciAgron/article/view/2273>. Acesso em: 24 dez. 2011.

ALUJA, M. et al. Seasonal population fluctuations and ecological implications for management of Anastrepha fruit flies (Diptera: Tephritidae) in commercial mango orchards in Southern Mexico. Journal of Economic Entomology, v.89, n.3, p.654-667, 1996.
BOMFIM, D.A. et al. Biodiversidade de moscas-das-frutas (Diptera: Tephritoidea) em matas nativas e pomares domésticos de dois municípios do Estado do Tocantins, Brasil. Revista Brasileira de Entomologia, v.51, n.2, p.217-223, 2007. Disponível em: <http://www.scielo.br/ s cielo.php? script $=$ sci_art text \& pid=S 0085 $56262007000200012 \& \operatorname{lng}=\mathrm{en}>$. Acesso em: $24 \mathrm{dez} .2011$. doi: http://dx.doi.org/10.1590/S0085-56262007000200012.

CRUZ, G.C.F. Alguns aspectos do clima dos Campos Gerais. In: MELO, M.S. et al. Patrimônio natural dos Campos Gerais do Paraná. Ponta Grossa: UEPG, 2007. 230p.

FEHN, L.M. Coleta e reconhecimento de moscas das frutas em região metropolitana de Curitiba e Irati, Paraná, Brasil. Anais da Sociedade Entomológica do Brasil, v.10, n.2, p.209238, 1981 .

FEHN, L.M. Levantamento da ocorrência de moscasdas-frutas Diptera: Tephritidae e Lonchaeidae, em pessegueiro, na área metropolitana de Curitiba e região de Irati-Paraná. 1977. 177f. Dissertação (Mestrado em Entomologia) - Universidade Federal do Paraná, PR.

GARCIA, F.R.M. Moscas-das-frutas (Diptera: Tephritidae) do estado do Paraná, Brasil. Acta Ambiental Catarinense, v.2, n.1, p.35-40, 2003.

GARCIA, F.R.M. et al. Análise faunística de espécies de moscasdas-frutas (Diptera: Tephritidae) na região oeste de Santa Catarina, Brasil. Neotropical Entomology, v.32, n.3, p.421426, 2003. Disponível em: <http://www.aba-agroecologia.org.br/ ojs2/index.php/cad/article/view/2919/2480>. Acesso em: 24 dez, 2011. doi: http://dx.doi.org/10.1590/S1519566X2003000300006. 
GUIMARÃES, J.A. et al Análise faunística de espécies de Tephritidae em pomar comercial de goiaba em Juazeiro do Norte, Ceará. In: CONGRESSO BRASILEIRO DE FRUTICULTURA, 20., 2008, Vitória, ES. Livro de resumos... Vitória: INCAPER, 2008. $5 \mathrm{p}$

HAMMER, $\varnothing$. et al. PAST: paleontological statistics software package for education and data analysis. Palaeontologia Electronica, 4. 2001. Disponível em: <http://www.nhm.uio.no/ norges/past/download.html>. Acesso em: 09 fev. 2011.

MALAVASI, A. et al. Biogeografia. In: MALAVASI, A.; ZUCCHI, R.A. Mosca-das-frutas de importância econômica no Brasil: conhecimento básico e aplicado. Ribeirão Preto: Holos, 2000. Cap.10, p.93-98.

MORAES, R.C.B. et al. Software para análise faunística ANAFAU. In: SIMPÓSIO DE CONTROLE BIOLÓGICO, 8. São Pedro, SP. Resumos... Piracicaba: ESALQ/USP, 2003. p.195.

SEAB. (Secretaria da Agricultura e do Abastecimento do Paraná). 2009. Disponível em: <http://www.seab.pr.gov.br/>. Acesso em: 27 abr. 2010

SCOZ, P. L. et al. Avaliação de atrativos alimentares e armadilhas para o monitoramento de Anastrepha fraterculus (Wiedemann, 1830) (Diptera: Tephritidae) na cultura do pessegueiro (Prunus persica (L.) Batsh). Idésia, v.24, n.2. p.7-13, 2006

SILVEIRA NETO, S. et al. Manual de ecologia dos insetos Piracicaba: Ceres, 1976. 419p.

URAMOTO, K. Biodiversidade de moscas-das-frutas do gênero Anastrepha (Diptera: Tephritidae) no campus Luiz de Queiroz, Piracicaba, São Paulo. 2002. 85f. Dissertação (Mestrado em Entomologia) - Escola Superior de Agricultura "Luiz de Queiroz", Universidade de São Paulo, SP.

ZILLI, G.; GARCIA, F.R.M. Análise faunística e flutuação populacional de moscas-das-frutas (Diptera: Tephritidae) em pomar de Citrus sinensis no município de Chapecó, Santa Catarina. Biodiversidade Pampeana, v.8, n.1, p.39-45, 2010. Disponível em: <http://revistaseletronicas.pucrs.br/ojs/ index.php/biodiversidadepampeana/article/viewFile/8064/ 6008>. Acesso em: 24 dez. 2011.

ZUCCHI, R.A. Taxonomia. In: MALAVASI, A.; ZUCCHI, R.A. Mosca-das-frutas de importância econômica no Brasil: conhecimento básico e aplicado. Ribeirão Preto: Holos, 2000a. Cap.1, p.13-24.

ZUCCHI, R.A. Espécies de Anastrepha, sinonímias, plantas hospedeiras e parasitóide. In: MALAVASI, A.; ZUCCHI, R.A Moscas-das-frutas de importância econômica no Brasil: conhecimento básico e aplicado. Ribeirão Preto: Holos, 2000b. Cap.4, p.41-48.

ZUCCHI, R.A. Mosca-do-mediterrâneo, Ceratitis capitata (Diptera: Tephritidae). In: VILELA, E.F. et al. Histórico impacto das pragas introduzidas no Brasil. Ribeirão Preto: Holos, 2001. p.15-22.

ZUCCHI, R.A. Diversidad, distribución y hospederos del género Anastrepha en Brasil. In: ORTIZ-HERNANDÉZ, V. Moscas de la fruta en Latinoamérica (Diptera: Tephritidae): diversidad, biología y manejo. Distrito Federal, México: S y G Editores, 2007. p.77-100. 